\title{
Interactive comment on "Spatial and seasonal effects on the delayed ionospheric response to solar EUV changes” by Erik Schmölter et al.
}

\section{Erik Schmölter et al.}

erik.schmoelter@dlr.de

Received and published: 15 August 2019

Thank you so much for the comments and suggestions to our manuscript! We will revise the manuscript according to your advice.

1. In Figure 3, the authors compared the correlation coefficients and the time delay retrieved from two datasets by fixing local time or fixing location. The authors should note that the time delay of ionosphere to the solar EUV flux change depends on solar local time. The time delay inferred from fixed location dataset can be partly considered as the averaged delay at different local times. The authors should point out this issue.

Answer: We will clarify the difference between local time and fixed location analysis as suggested. 
"The delayed ionospheric response to solar EUV radiation depends on the solar local time and the calculated results for fixed locations can be understood as a mean ionospheric delay for different local times. The local time approach would be preferred for this reason. Nevertheless, the analysis with fixed local time is not used in the further analysis, since the extracted time series from the IGS TEC maps relies less on measurements considering areas with few or no ground stations."

2. The time delay of ionospheric parameters is the key in this analysis. The difference between the time delay by using the 2 methods is greater than 4 hours. How about the uncertainty of the obtained time delay? In addition, the diurnal variation of ionospheric parameters may affect the calculation of time delay. They can provide the time delay by removing the diurnal variation in Figure 3.

Answer: We will add an explanation of the mean difference (approximately 3.15 hours) between both approaches and characterized the uncertainty.

"The two different approaches have a mean variance of approximately 3.15 hours, which accounts for an uncertainty of approximately $16.04 \%$ in the ionospheric delay calculation. This is an acceptable impact of the diurnal variation on the trend of the delay for characterizing temporal and spatial changes."

The time delay can be provided without the diurnal variation, but the available approaches don't improve the process or even have a negative impact. Removing the diurnal variation with a band-stop filter doesn't remove the diurnal variation completely and there is no improvement for the correlation and reliability of the delay (Schmölter et al., 2018). Calculating daily mean values for TEC or foF2 doesn't allow a delay analysis on hourly resolution, since that would require interpolation back to the high resolution and this in turn has a huge impact on the delay calculation. In general, an improvement of the correlation coefficients (e.g. calculating daily means) doesn't grant a more reliable or precise delay calculation. We decided against filters or changes on the signal, acknowledge the impact of the diurnal variation and focus on features of the

Interactive comment
Printer-friendly version

Discussion paper 
ionospheric delay, which are not defined by small scale changes. In addition, the calculated value range and features of the delay trend fit very well with results of preceding studies.

3. Is the time delay reliable as the correlation coefficient is less than 0.4 ?

Answer: The results are statistically significant due to the big sample size (90 days on hourly resolution) and, as shown by Figures 2 and 3 , the relative trend of correlation coefficients and delay is not changed in different approaches. For example using fixed local times gives much higher correlation coefficients and the resulting annual variation of the delay is present.

We will clarify the reliability in the manuscript as mentioned in the reply to comment 2.

4. How do they calculate the $\mathrm{Kp}$ index, the red line in Figure 4?

Answer: We will clarify the description of Figure 4. The Kp-index data are shown in weekly resolution with the red line, because the trend on hourly or daily resolution doesn't give a meaningful overview for the long-term changes due to the much stronger short-term variations. A description for the calculation of the smoothed trends will be added as well.

"The transparent red lines or dots show the raw data: Kp-index in weekly resolution (a), correlation coefficients between EUV and TEC (b) and delays between EUV and TEC (c) in hourly resolution. The black lines show the smoothed weekly means to present the overall trend (running mean with window size of 10 days)."

5. In Figure 4, the $\mathrm{Kp}$ index, the correlation coefficient and time delay show similar decreases during the end of each year. The authors indicated that the lower correlation coefficient and time delay should be related to the corresponding lower geomagnetic activity. Why the correlation coefficient is lower when the geomagnetic disturbance $(\mathrm{Kp})$ is lower?

Answer: The explanation of annual variations of the correlation coefficients and iono-

Interactive

comment 
spheric delay with geomagnetic activity is difficult and requires modeling efforts in the future. The topic introduces a lot of complexity due to the various ways geomagnetic activity impacts the ionospheric state. An explanation could be the global F2 layer ionization due to geomagnetic activity (Lal, C. ( 1992), Global F2 layer ionization and geomagnetic activity, J. Geophys. Res., 97( A8), 12153-12159, doi:10.1029/92JA00325.). As already suggested in the manuscript, analyses for longer time periods are required to further explain this relation and the processes behind it.

6. In Figures 11 and 12, the time delay generally does not change with latitude in winter. Whereas, during winter time the correlation coefficient is nearly 0 as seen in Figure 4. Therefore, the absolute values of the correlation coefficient should be provided in Figures 11 and 12.

Answer: We will add the absolute correlation coefficients to Figures 11 and 12 and modify the captions accordingly.

"Figure 11: Map of the delay of TEC with respect to EUV in summer (May to August) and winter (November to February) within the time period from 2011 to 2014 . The delay varies between $\approx 18.6$ and $\approx 21.7$ hours. The hatched regions on the map represent significantly greater (upper left to lower right fill) or smaller (upper right to lower left fill) correlations compared to the average of each map ( \pm one standard deviation). The absolute correlation coefficient is $\approx 0.28$ in summer and $\approx 0.17$ in winter."

"Figure 12: Time series of the delay of TEC with respect to EUV as an epoch plot for the mid-latitudes covering Europe within the time period from 2011 to 2013 . The delay varies between $\approx 11.3$ and $\approx 23.1$ hours. The absolute correlation coefficient is $\approx 0.21$ during the period."

Interactive comment on Ann. Geophys. Discuss., https://doi.org/10.5194/angeo-2019-91, 2019.

Interactive

comment

Discussion paper 